\title{
Correlation between growth kinetics and nanoscale resistive switching properties of $\mathrm{SrTiO}_{3}$ thin films
}

Ruth Muenstermann, Tobias Menke, Regina Dittmann, Shaobo Mi, Chun-Lin Jia, Daesung Park, and Joachim Mayer

Citation: Journal of Applied Physics 108, 124504 (2010);

View online: https://doi.org/10.1063/1.3520674

View Table of Contents: http://aip.scitation.org/toc/jap/108/12

Published by the American Institute of Physics

\section{Articles you may be interested in}

Grain boundaries as preferential sites for resistive switching in the $\mathrm{HfO}_{2}$ resistive random access memory structures

Applied Physics Letters 100, 123508 (2012); 10.1063/1.3697648

Realization of regular arrays of nanoscale resistive switching blocks in thin films of $\mathrm{Nb}$-doped $\mathrm{SrTiO}_{3}$ Applied Physics Letters 93, 023110 (2008); 10.1063/1.2959074

Leakage current through the poly-crystalline $\mathrm{HfO}_{2}$ : Trap densities at grains and grain boundaries Journal of Applied Physics 114, 134503 (2013); 10.1063/1.4823854

Resistive switching mechanism of $\mathrm{TiO}_{2}$ thin films grown by atomic-layer deposition Journal of Applied Physics 98, 033715 (2005); 10.1063/1.2001146

Reproducible switching effect in thin oxide films for memory applications Applied Physics Letters 77, 139 (2000); 10.1063/1.126902

Note: Electrical resolution during conductive atomic force microscopy measurements under different environmental conditions and contact forces

Review of Scientific Instruments 81, 106110 (2010); 10.1063/1.3491956

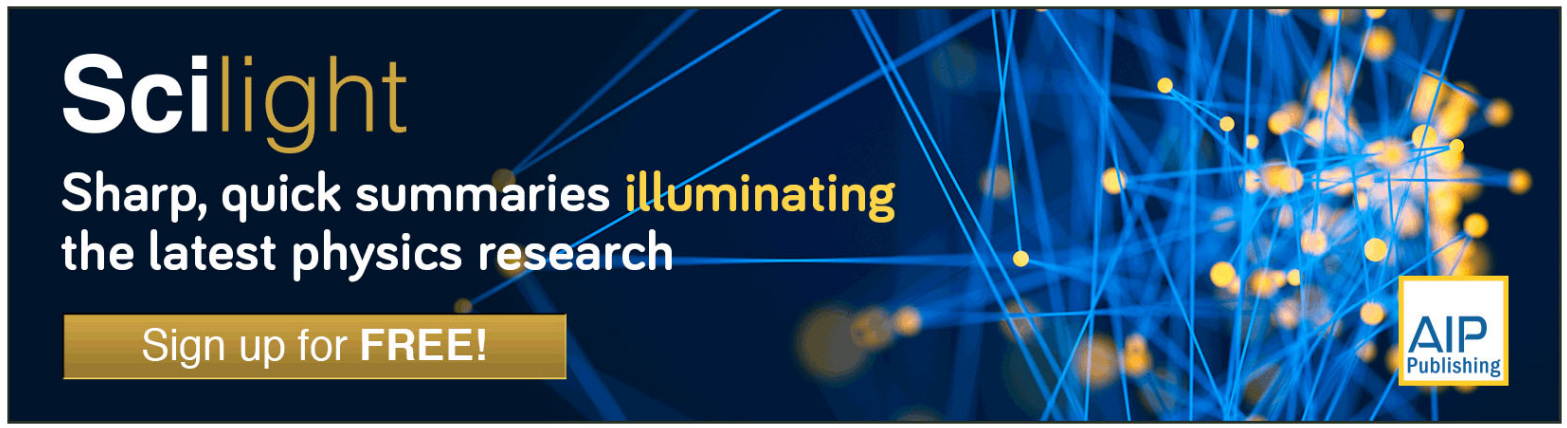




\title{
Correlation between growth kinetics and nanoscale resistive switching properties of $\mathrm{SrTiO}_{3}$ thin films
}

\author{
Ruth Muenstermann, ${ }^{1, a)}$ Tobias Menke, ${ }^{1}$ Regina Dittmann, ${ }^{1}$ Shaobo $\mathrm{Mi},{ }^{2}$ Chun-Lin $\mathrm{Jia},{ }^{2}$ \\ Daesung Park, ${ }^{3}$ and Joachim Mayer ${ }^{3}$ \\ ${ }_{1}^{1}$ Institute for Electronic Materials, Research Center Juelich, 52425 Juelich, Germany \\ ${ }^{2}$ Ernst Ruska-Centre (ER-C) for Microscopy and Spectroscopy with Electrons, Research Center Juelich, \\ 52425 Juelich, Germany \\ ${ }^{3}$ Central Facility for Electron Microscopy (GFE), RWTH Aachen University, 52074 Aachen, Germany
}

(Received 19 August 2010; accepted 28 October 2010; published online 22 December 2010)

\begin{abstract}
We deliberately fabricated $\mathrm{SrTiO}_{3}$ thin films deviating from ideal stoichiometry and from two-dimensional layer-by-layer growth mode, in order to study the impact of well pronounced defect arrangements on the nanoscale electrical properties. By combining transmission electron microscopy with conductive-tip atomic force microscopy we succeeded to elucidate the microstructure of thin films grown by pulsed laser deposition under kinetically limited growth conditions and to correlate it with the local electrical properties. $\mathrm{SrTiO}_{3}$ thin films, grown in a layer-by-layer growth mode, exhibit a defect structure and conductivity pattern close to single crystals, containing irregularly distributed, resistive switching spots. In contrast to this, Ti-rich films exhibit short-range-ordered, well-conducting resistive switching units. For Ti-rich films grown in a kinetically more restricted island growth mode, we succeeded to identify defective island boundaries with the location of tip-induced resistive switching. The observed nanoscale switching behavior is consistent with a voltage driven oxygen vacancy movement that induces a local redox-based metal-to-insulator transition. Switching occurs preferentially in defect-rich regions, that exhibit a high concentration of oxygen vacancies and might act as easy-diffusion-channels. (C) 2010 American Institute of Physics. [doi:10.1063/1.3520674]
\end{abstract}

\section{INTRODUCTION}

Transition metal oxides have attracted considerable attention over the last decades due to their large variety of functional properties, e.g., high $T_{c}$ superconductivity, high permittivity, or multiferroicity. The typical perovskite lattice of these materials allows for a broad range of point and extended defects which strongly influence the electronic properties. The most prominent example are oxygen vacancies that generally act as donors. As a result, the band insulator $\mathrm{SrTiO}_{3}$ exhibits metallic or even superconducting properties under donor impurity doping or at high oxygen vacancies concentrations. ${ }^{1,2}$ In thin film form, transition metal oxides are suitable for various electronic device applications and moreover, offer new functionalities, e.g., new order parameters in ferroelectric superlattices ${ }^{3}$ or the formation of a twodimensional (2D) electron gas at $\mathrm{SrTiO}_{3} / \mathrm{LaAlO}_{3}$ heterointerfaces. ${ }^{4}$ However, due to the nonequilibrium conditions inherent to most vapor deposition techniques, thin films exhibit a different defect structure than bulk samples, fabricated under equilibrium conditions, which might be either beneficial or deleterious for their functional properties. ${ }^{5}$ In ferroelectric materials, defects control the local polarization stability, act as pinning sites for domain wall motion and nucleation sites for polarization reversal. ${ }^{6}$ Therefore, the control over defect density and distribution is a key for engineering the functional properties of ferroic thin films. Moreover, extended defects, e.g., dislocations, are considered to act as

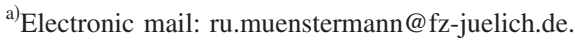

nanoscale functional units in resistive switching $\mathrm{SrTiO}_{3}$ single crystals and thin films, which are under consideration for future nonvolatile memories with terabit density. ${ }^{7}$ These, so called memristive elements, ${ }^{8,9}$ require a considerable amount of defects to sustain their functionality. ${ }^{10}$ Therefore, the growth of thin films with ultimate crystalline perfection is counterproductive for the realization of reliable resistive switching devices. As a results of this change in paradigm, a breakthrough in the field of resistive switching devices can be expected if the realization of thin films with tailored defect structure becomes feasible. On the way toward the fabrication of defect engineered memristive thin films, it is inevitable to clarify the switching properties of specific single defects or well defined defect conglomerations.

In this work, we investigated the local conductivity and the local resistive switching properties in thin films with specific defect structure. By varying the deposition conditions during pulsed laser deposition (PLD), we modified the growth kinetics and thereby the local distribution of defects. Starting with thin films grown in a defined 2D layer-by-layer mode, we deliberately fabricated thin films in a threedimensional (3D) growth mode. Detailed investigations by x-ray diffraction (XRD), transmission electron microscopy (TEM), atomic force microscopy (AFM), and conductive tipAFM (LC-AFM) are combined to elucidate the correlation between the microstructure and the local conductive properties of thin films grown under kinetically limited growth conditions. 
TABLE I. Parameters used for PLD growth of 2 at. \% Nb-doped $\mathrm{SrTiO}_{3}$ and 1 at. \% Fe-doped $\mathrm{SrTiO}_{3}$ thin films.

\begin{tabular}{lcccc}
\hline \hline Parameter set & I & II & III & IV \\
Growth type & 2D & & 700 & \\
Temperature $\left({ }^{\circ} \mathrm{C}\right)$ & & & 70 & \\
Pressure & $0.25 \mathrm{mbar}_{2}$ & $0.25 \mathrm{mbar} \mathrm{O}_{2}$ & $10^{-4} \mathrm{mbar} \mathrm{Ar}$ & $0.25 \mathrm{mbar} \mathrm{O}_{2}$ \\
Frequency $(\mathrm{Hz})$ & 5 & 30 & 10 & 10 \\
Laser fluence $\left(\mathrm{J} / \mathrm{cm}^{2}\right)$ & 0.8 & 0.8 & 2 & 2 \\
Growth rate $(\AA / \mathrm{s})$ & 0.47 & 2.84 & 7.67 & 3.33 \\
Substrate & & 1 at. \% Nb-doped $\mathrm{SrTiO}_{3}$ & \\
\hline \hline
\end{tabular}

\section{EXPERIMENTAL}

1 at. \% Nb-doped $\mathrm{SrTiO}_{3}(001)$ oriented single crystal with a nominal lattice constant of $3.905 \AA$ (Ref. 11) were used as substrates. Due to their metallic conductivity, the substrates serve as a bottom electrode in subsequent resistive switching measurements. Prior to thin film deposition, the substrates were annealed for approximately $4-6 \mathrm{~h}$ at $1000{ }^{\circ} \mathrm{C}$ in air to ensure a defined, flat surface, exhibiting a step-terrace structure.

Thin films were grown by PLD using a $\mathrm{KrF}$ excimer laser $(248 \mathrm{~nm})$ and ceramic targets. Undoped $\mathrm{SrTiO}_{3}$, 2 at. \% Nb-doped $\mathrm{SrTiO}_{3}$, and 1 at. \% Fe-doped $\mathrm{SrTiO}_{3}$ ceramic targets were employed for thin film deposition. Table I gives a summary of the used deposition parameter sets.

In order to investigate the local nature of the conductivity and the switching behavior of the samples we performed LC-AFM measurements of all films. All measurements were carried out with a Jeol JSPM 4210 microscope employing a PtIr-coated AFM tip. The chamber containing the sample was evacuated to a background pressure of $10^{-5} \mathrm{mbar}$ in order to reduce the influence of surface adsorbates. The current compliance was set to $1 \mu \mathrm{A}$ since higher currents can cause a delamination of the PtIr-coating. The lateral electrical resolution of the tip was demonstrated to be in the order of a few nanometers, which is consistent with theoretical estimations of the effective contact area between tip and sample. ${ }^{7,12,13}$

The applied voltage is always given with respect to the tip. By choosing an appropriate tip voltage, the AFM tip can either be used as a passive readout tool (Secs. III-V) or as a mobile, active switching electrode (Sec. VI). Finite element simulations show that the main part of a voltage applied between tip and sample drops directly below the tip. ${ }^{14}$ Any tip induced resistive switching therefore takes place within the surface near region of the sample.

\section{LAYER-BY-LAYER GROWTH}

Using the deposition parameters given in set I in Table I, $50 \mathrm{~nm}$ thick films of 1 at. \% Fe-doped $\mathrm{SrTiO}_{3}$ were grown. Figure 1 shows the XRD overview scan of such a sample as well as a close-up of the region around the $\mathrm{SrTiO}_{3}(002)$ peak. The overview spectrum exhibits only three very sharp peak doublets, belonging to single crystalline $\mathrm{SrTiO}_{3}$, caused by the substrate. A closer look at the (002) region of the spectrum and a comparison with a spectrum taken from a bare substrate (without any film on top) proves that the film structure is identical to the substrate structure [Fig. 1(b)], as no difference between the two spectra can be detected.

This lack of a lattice expansion is a sign for a stoichiometric $\mathrm{SrTiO}_{3}$ thin film with a Ti/Sr ratio close to 1 , since nonstoichiometries result in a significant lattice expansion (Schlom). Ohnishi et al. as well as other groups have shown that for PLD grown $\mathrm{SrTiO}_{3}$ thin films the stoichiometry is directly determined by the laser fluence: ${ }^{15-18}$ a low laser fluence results in $\mathrm{Sr}$ rich films, while a high fluence produces $\mathrm{Ti}$ rich films. While $\mathrm{Sr}$ and $\mathrm{Ti}$ vacancies are therefore negligible, subsequent electrical measurements indicate, that the films contain a significant amount of oxygen vacancies (not shown here), that are not detectable in a XRD scan.

The AFM tapping mode surface scan of such a film is shown in Fig. 2(a). The surface is very smooth and step terraces with a height of one atomic layer can be seen. This morphology indicates that the film is grown in a 2D growth mode, preserving the step terraces of the underlying substrate. Cross-sectional TEM images confirm that this growth mode results in a coherent growth and a low defect density [Fig. 2(d)].

Finally, a behavior close to single crystals can also be found in the conductive AFM scans [Figs. 2(b) and 2(c)]. The conductivity image displays many small and very well conducting spots that are randomly distributed within a poorly conducting background. Comparing this pattern to the conductive AFM patterns seen for $\mathrm{SrTiO}_{3}$ single crystals reveals almost identical behavior. ${ }^{7}$ The individual conducting spots have a diameter of only $1-2 \mathrm{~nm}$ and were suggested to be identified with extended defects, in particular dislocations, that stretch across the sample and intersect with the surface. First principles calculations indicate an increased density of states (DOS) at the lower conduction band edge within these
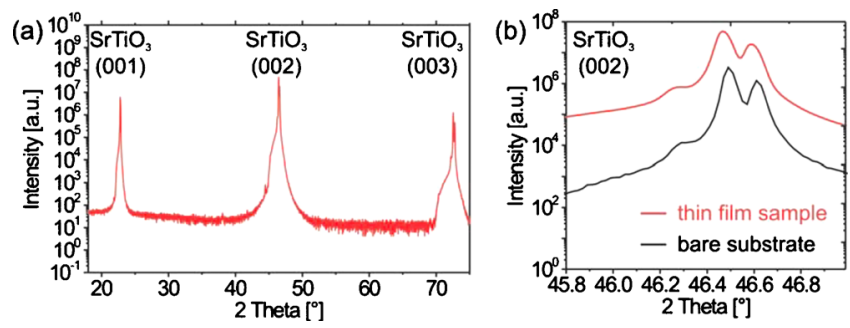

FIG. 1. (Color online) (a) XRD overview scan of a $50 \mathrm{~nm}$ thick Fe-doped $\mathrm{SrTiO}_{3}$ film grown under deposition conditions I. The film structure has merged completely with the substrate structure, leaving only the $\mathrm{SrTiO}_{3}$ single crystal substrate peaks visible. (b) A magnified look at the $\mathrm{SrTiO}_{3}$ (002) region of the film shown in (a) and a separate XRD scan of a bare substrate show no discernable structural difference. 

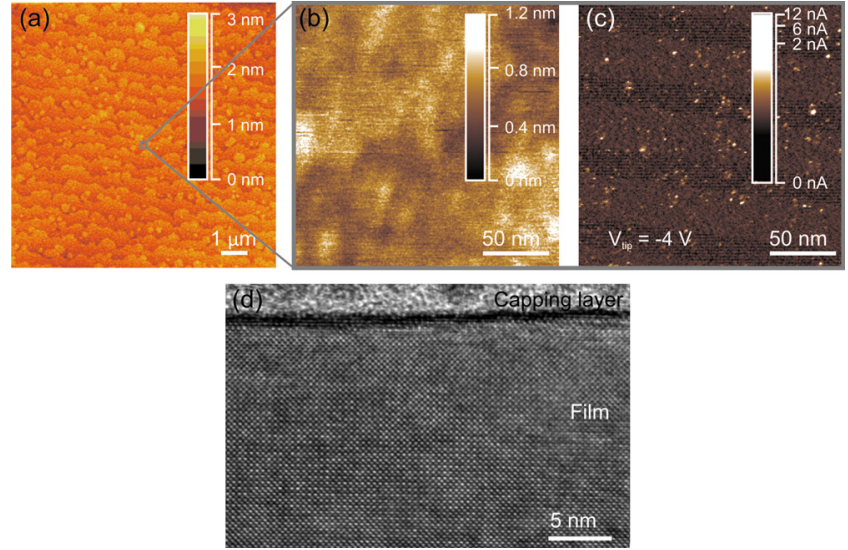

FIG. 2. (Color online) (a) Tapping mode AFM surface scan of a $50 \mathrm{~nm}$ thick Fe-doped $\mathrm{SrTiO}_{3}$ film grown under deposition conditions I. The surface is very smooth and shows step terraces with a height of one atomic layer. (b) Conductive AFM surface and (c) conductivity scan. The strongly shrunk scan size as well as the reduced lateral resolution of the conductive AFM contact mode scan lead to a different perception of the topography in part (b) as opposed to part (a). The conductivity is inhomogeneous and has a pattern comparable to that of $\mathrm{SrTiO}_{3}$ single crystals (Ref. 7). (d) The corresponding TEM data show a flat, 2D layer-by-layer growth.

dislocations and a corresponding increased electronic conductivity along the dislocation. ${ }^{7}$ However, due to their low density and irregular distribution, we were unable to directly identify dislocations as conducting filaments in our high resolution transmission electron microscopy investigations.

Overall it can be stated that the growth conditions in set I of Table I provide flat, step-terraced $\mathrm{SrTiO}_{3}$ films grown in a $2 \mathrm{D}$ mode that behave similarly to single crystals and have a $\mathrm{Ti} / \mathrm{Sr}$ ratio close to 1 .

\section{IMPURITY DOPING}

In general chemical impurity doping has an important influence on the macroscopic conductivity of a sample. P-type $\mathrm{Fe}$-doped $\mathrm{SrTiO}_{3}$ single crystals behave different from n-type $\mathrm{Nb}$-doped $\mathrm{SrTiO}_{3}$ single crystals in terms of electronic properties and chemical compensation mechanisms. ${ }^{19}$ In the case of thin film growth, however, the prepared samples are usually far away from the stoichiometry of a single crystal. Other effects - such as a growth related oxygen deficiency or a shift in the Ti/Sr ratio-play an important role and can potentially overrule the external chemical doping. ${ }^{16}$

In order to determine the influence of chemical impurity doping on the structure and local conductivity of our PLD grown thin films, a 2 at. \% Nb-doped $\mathrm{SrTiO}_{3}$ thin film and a 1 at. \% Fe-doped $\mathrm{SrTiO}_{3}$ thin film were prepared under the deposition conditions described in set II in Table I and checked with conductive AFM. The deposition parameters are nearly identical to the previously chosen layer-by-layer case. The only difference is an increased growth rate due to a raised laser frequency.

The topography of both samples is shown in Figs. 3(a) and 3(d). Both of them show comparable surface features with a root mean square $(\mathrm{rms})$ roughness of about $0.2 \mathrm{~nm}$. Step terraces similar to the previous layer-by-layer growth can be seen. Focusing onto a single terrace, however, as
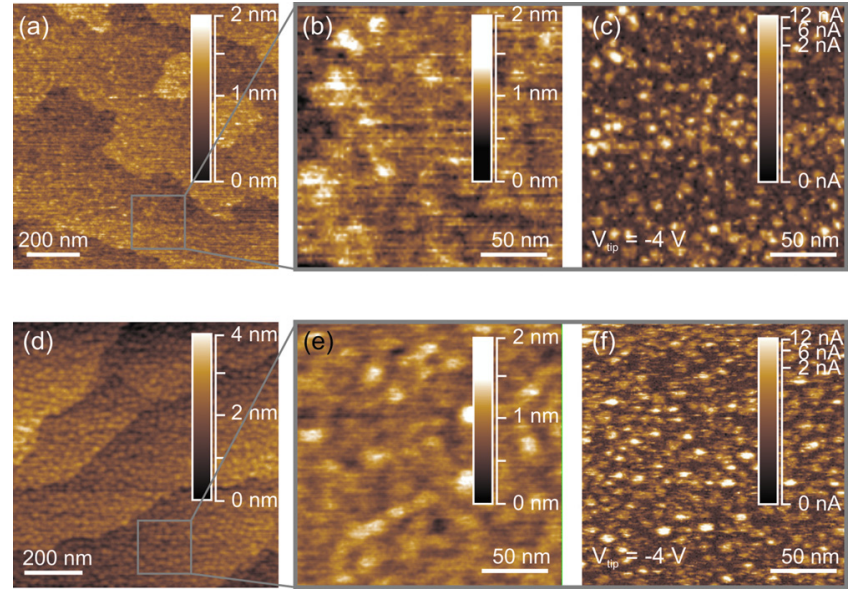

FIG. 3. (Color online) (a) Topography of a 2 at. $\%$ Nb-doped $\mathrm{SrTiO}_{3}$ thin film grown under deposition conditions II of Table I. (b) The conductive AFM topography and (c) conductivity scan were recorded with a smaller scan range, focusing onto a single terrace. (d) Overview topography, (e) magnified topography, and (f) conductivity of a 1 at. \% Fe-doped $\mathrm{SrTiO}_{3}$ thin film grown under identical conditions (set II of Table I). Neither morphology nor local conductivity are significantly altered by the change in dopant type.

shown in Figs. 3(b) and 3(e), reveals a hilly substructure and a deterioration in the terrace smoothness. A corresponding pattern can be seen in the current images [Figs. 3(c) and 3(f)]. They exhibit a pattern of enlarged conducting units. The overall change in surface morphology and local conductivity compared to Fig. 2 can be related to the increased deposition rate and will be discussed in more detail in the following sections.

Concerning the comparison of $\mathrm{Fe}$ - and $\mathrm{Nb}$-doped samples, no difference can be observed on a local scale. The structure and conductivity appear to be dominantly determined by the identical thin film deposition conditions. It is interesting to note, that this dominance of the deposition conditions over the chemical impurity doping is also reflected on a macroscopic level. Checking the macroscopic conductivity of these samples, reveals that our $\mathrm{Fe}$-doped $\mathrm{SrTiO}_{3}$ films generally exhibit $n$-type semiconducting behavior. ${ }^{20}$ The specific growth and defect structure of those films obviously allows to accommodate a significant amount of oxygen vacancies. Previous studies on $\mathrm{SrTiO}_{3}$ single crystals suggest that interfaces ${ }^{21}$ and extended defects ${ }^{22}$ are preferential places for oxygen vacancies. These inherently oxygendeficient defects outweigh the background Fe acceptor doping and result in a dopant-independent n-type behavior.

Due to the complex nature of PLD grown thin films, the overall conductive properties are therefore strongly dominated by the growth related specific defect structure and stoichiometry and external impurity doping plays a minor role.

\section{3D GROWTH}

In order to access different types of film growth than the previously described layer-by-layer growth, extreme cases of deposition conditions were chosen (set III and IV in Table I), producing films of widely different defect structures grown in a $3 \mathrm{D}$ way. 

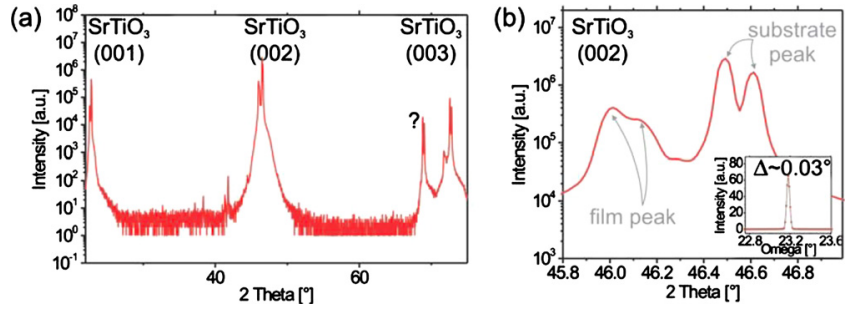

FIG. 4. (Color online) (a) XRD overview scan of a $200 \mathrm{~nm}$ thick 2 at. $\%$ $\mathrm{Nb}$-doped $\mathrm{SrTiO}_{3}$ film grown under deposition conditions III. $\mathrm{SrTiO}_{3}(001)$, (002), and (003) peaks are visible for both, the thin film and substrate, corresponding to lattice constants of $3.943 \AA$ in case of the film and $3.903 \AA$ in case of the substrate. (b) A magnified look at the $\mathrm{SrTiO}_{3}$ (002) region showing the difference in peak position and lattice constant for film and substrate. The respective rocking curve for the film peak is shown in the inset.

The laser fluence was set to its maximum value (approximately $2 \mathrm{~J} / \mathrm{cm}^{2}$ ) driving the films into a clearly nonstoichiometric, Ti rich conditions. ${ }^{15-18}$ In addition the laser frequency has been doubled. Combined with the raised fluence, this leads to a significantly increased deposition rate and a further leaning toward a strongly kinetically limited growth. A subsequent variation in deposition pressure between set III and IV highlights the connection between pressure and growth kinetics and their implications for thin film growth.

\section{A. Coherent 3D growth}

Figure 4 shows XRD spectra of a $200 \mathrm{~nm}$ thick 2 at. \% $\mathrm{Nb}$-doped $\mathrm{SrTiO}_{3}$ film grown under deposition conditions III. While, compared to the previous case of layer-by-layer grown $\mathrm{Fe}$-doped $\mathrm{SrTiO}_{3}$, the influence of the change in dopant material from $\mathrm{Fe}$ to $\mathrm{Nb}$ is less important for the growth structure (see Sec. IV), the change in deposition parameters has caused a significant difference in the spectra: with all three substrate $\mathrm{SrTiO}_{3}$ peaks still visible, a closer look at the peak regions [Fig. 4(b)] reveals that in addition to the sharp substrate peaks, clearly shifted and more broadened film peaks can be seen. Those peaks still represent a perovskite $\mathrm{SrTiO}_{3}$ structure but the shift in peak positions indicates an expanded lattice constant: the peak positions of the film peaks correspond to a lattice constant of $3.943 \AA$, while the substrate peaks represent a substrate lattice constant of 3.903 $\AA$ [which is nearly identical to the literature bulk value of $3.903 \AA$ (Ref. 11)]. The film is still c-axis oriented, epitaxial and has very good crystallinity [as indicated by the rather small Rocking curve width of $0.03^{\circ}$ shown in the inset of Fig. 4(b)].

The expanded lattice constant is caused by a shift in the $\mathrm{Ti} / \mathrm{Sr}$ ratio due to the relatively high laser fluence during deposition. ${ }^{16}$ A comparison with literature data suggests a $\mathrm{Ti} / \mathrm{Sr}$ ratio of roughly $1.5,{ }^{18}$ which is in good agreement with element concentrations determined by x-ray photoelectron spectroscopy (XPS) for these films. ${ }^{23}$ While 1.5 may not be a quantitatively accurate value, it clearly indicates a Ti excess or an equivalent increase in $\mathrm{Sr}$ vacancies. Since $\mathrm{Sr}$ vacancies are charged, electrostatic repulsion causes an outwards movement of the atoms surrounding a vacancy and an isotropic expansion of the lattice resulting in an in-plane lattice
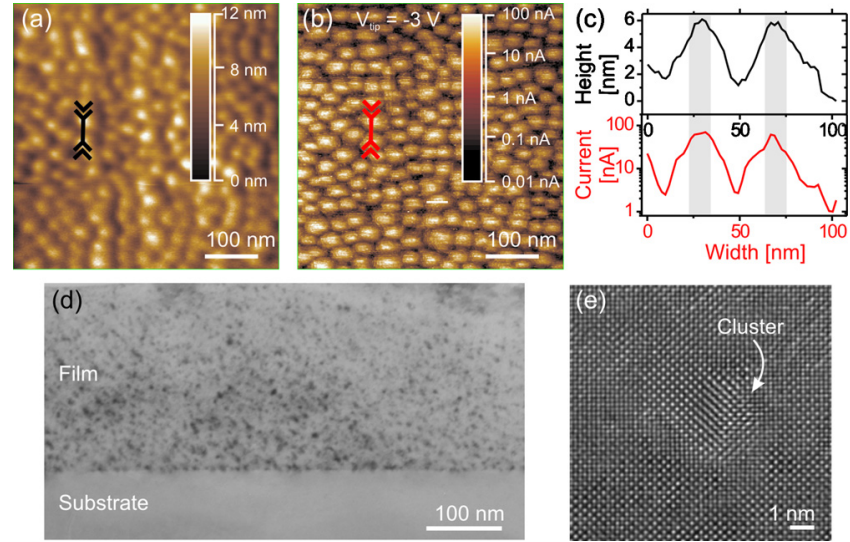

FIG. 5. (Color online) (a) Topography and (b) current image of a 2 at. \% $\mathrm{Nb}$-doped $\mathrm{SrTiO}_{3}$ film grown under deposition conditions III. The surface consists of small, circular hills with a conductive center and nonconducting boundaries. (c) This correlation is visualized by two line scans taken at the same position within topography and current image. [(d) and (e)] The defect structure was checked by cross-sectional TEM and exhibits a dense distribution of defect-rich nanoclusters.

mismatch to stoichiometric $\mathrm{SrTiO}_{3}$ of the order of $1 \% .^{24}$ As a result, the Ti-rich $\mathrm{SrTiO}_{3}$ grows compressively strained on stoichiometric $\mathrm{SrTiO}_{3}$ single crystals, resulting in the observed elongation of the c-axis and strong deviations from homoepitaxial layer-by-layer growth. This growth parameter induced transition from a 2D, layer-by-layer mode to a different, 3D growth type is reflected in both the morphology and conductivity as observed with conductive AFM in Figs. 5(a)-5(c). The topography in part (a) has no longer a flat, step-terrace structure but exhibits a dense array of roughly 30 $\mathrm{nm}$ wide and 1-4 $\mathrm{nm}$ high, round hills, having a certain degree of short-range ordering. The corresponding rms surface roughness lies at $1.78 \mathrm{~nm}$. The local current distribution [recorded at a tip voltage of $-3 \mathrm{~V}$, see Fig. 5(b)], is significantly changed as well and somehow reflects the hilly surface structure. A dense array of well-conducting units can be seen across a nonconducting background. A comparison between topography and current can be achieved by placing a line scan of the topography image, taken at the site indicated by the black line, above a line scan of the current image, taken at exactly the same position as indicated by a red line [Fig. 5(c)]. Both, topography and current, reveal a direct spatial correlation: the center of each surface hill coincides with the center of each well conducting unit, while the boundaries of the surface hills are less conductive regions.

While AFM probes only the surface of a sample, crosssectional TEM can be used to investigate deeper lying regions and to determine the defect structure. Figure 5(d) shows a cross-sectional overview of a film. While the film is grown in a coherent, epitaxial way, a dense distribution of small black spots can be seen across the film thickness. A magnification of these spots, as shown in Fig. 5(e), reveals the structure of the spots. The image contrast of the spots is related to the small nanoclusters with strong local lattice distortion. Analysis shows that the clusters have increased lattice constant in comparison with the perfect lattice of matrix. While the initial explanations for this increased lattice constant revolved around an increased $\mathrm{Nb}$-concentration within 
these clusters, ${ }^{25}$ subsequent energy dispersive x-ray spectroscopy scans showed no inhomogeneity in the $\mathrm{Nb}$ content. In view of the $\mathrm{Sr}$ deficient nature of our films a local enrichment in $\mathrm{Sr}$ vacancies is more plausible. Those vacancies can constitute the increased defect density within the clusters and at the same time account for the local expansion of the lattice constant (due to their electrostatic repulsion). Ohnishi et al. ${ }^{15}$ have described a similar defect structure of Sr vacancy rich inclusions in a related material.

Looking for a general picture of the growth structure of our films by combining all available data, the high laser fluence has led to a nonstoichiometry and lattice expansion and a heteroepitaxylike condition, while the combination of an extremely high growth rate and a low deposition pressure imposes complex kinetic limitations. This specific set of growth conditions results in a growth type previously described as "pseudo-2D island growth," 26 that is still 3D in character but has much smaller surface islands than the classical 3D Stranski-Krastanov or Volmer-Weber growth modes. A specific feature of this growth type is the uniform, circular shape of the surface islands, their dense arrangement and a certain degree of short-range ordering between them, as seen in Fig. 5(a). It has been suggested that the surface islands are related to threading dislocations intersecting with the film surface. ${ }^{26}$ There are three arguments in favor of this interpretation: on the one hand threading dislocations are the main extended defects in $\mathrm{SrTiO}_{3}$ thin films. ${ }^{27}$ On the other hand they are known to form a roughly regular network of dislocations with a surface density similar to that of the surface islands. ${ }^{27,28}$ Finally, if intersecting with the surface, a threading dislocation forms roughly pyramidal structures, that could be similar to the shape of an island. ${ }^{29}$ Concerning the relation between defect structure and conductivity it can be further speculated, that if the islands are related to threading dislocations, the center of an island or a dislocation should have a higher conductivity than the boundaries. This correlation would perfectly explain the observed conductivity pattern in Fig. 5(b). However, our TEM images do not allow to discern any threading dislocations since their visibility depends very critically on the observation direction. The only visible features in our current TEM data are the $\mathrm{Sr}$ vacancy rich inclusions that show no apparent connection with the surface morphology.

Combining all data it can be concluded that the films deposited under deposition conditions III are grown in a coherent, epitaxial but 3D way, have a $\mathrm{Ti}$ and defect-rich growth structure and a specific type of small, short-rangeordered, and well-conducting surface hills. However, a direct correlation between conductivity pattern and microstructural features could not be obtained for these samples.

\section{B. Island growth}

Keeping the same high laser fluence (and therefore, Ti/Sr ratio) and frequency as in Sec. V A but raising the deposition pressure results in altered growth kinetics and causes yet another type of growth and defect structure. Figure 6 shows
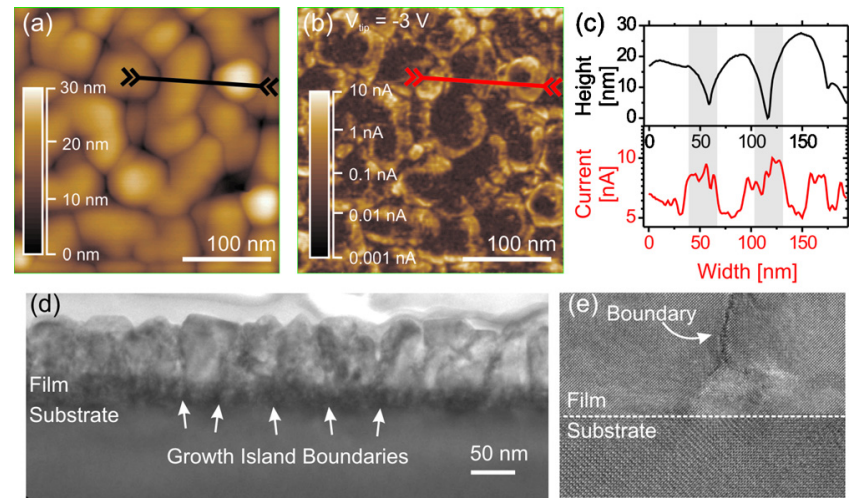

FIG. 6. (Color online) (a) Topography and (b) current image of a 2 at. \% $\mathrm{Nb}$-doped $\mathrm{SrTiO}_{3}$ film grown under an increased deposition pressure (deposition conditions IV). The surface consists of large islands with conducting boundaries. (c) The correlation between topography and conductivity is again visualized by two line scans taken. [(d) and (e)] The defect structure was checked by cross-sectional TEM. The film contains large growth islands, extending from the substrate to the surface, with defect-rich boundaries. These growth islands can be identified with the surface islands.

the topography, conductivity and defect structure of a 100 nm thick 2 at. \% Nb-doped $\mathrm{SrTiO}_{3}$ film grown under deposition conditions IV (see Table I).

The surface structure has completely changed toward a much rougher morphology (the rms roughness is approximately $5 \mathrm{~nm}$ ), showing slightly longish, 50-100 nm wide, and 10-20 nm high islands. The conductivity is also inhomogeneous but has a different pattern as in the previous, low pressure case (see Fig. 5). Instead of well-conducting units, well-conducting ringlike structures can be seen. Checking for a connection between topographic features and conductivity by placing two line scans at the same positions within topography and current image [Fig. 6(c)], both are again correlated with each other. However, in this case the correlation is exactly reversed to the previous case: the boundaries of each surface island are very well-conducting regions while the center is less conducting.

This specific conductivity distribution can be very well related to the films' defect structure as seen by TEM [Figs. 6(d) and 6(e)]. The overview image shows an epitaxial film, consisting of closely packed columnar structures, or growth islands, that extend from the substrate to the surface of the film. The boundaries of these islands can be seen as dark, defective regions. A few of them are marked exemplarily by white arrows and magnification of such an island boundary is shown in Fig. 6(e).

A similar columnar microstructure has been presented by He et $a l^{30}$ for $\mathrm{BaTiO}_{3}$ thin films. In their case, nearly stoichiometric $\mathrm{BaTiO}_{3}$ growth islands were surrounded by amorphous, Ti-rich boundaries. In our case, the Ti excess-imposed by the high laser fluence-has to stem from the island bulk and cannot be restricted to the boundaries since we observed an expanded lattice constant in the corresponding XRD spectra (not shown here).

The diameter of the columnar islands is of the order of $50 \mathrm{~nm}$ or more. Making a connection between TEM and AFM data, the surface islands seen by conductive AFM can clearly be identified with those columnar islands, extending down to the substrate and having defect-rich boundaries. 
The change in morphology and growth structure upon raising the deposition pressure can be well explained by the increased kinetic limitations, since the migration length is reduced by at least a factor of 4 when changing the pressure from set III to set IV in Table I. ${ }^{31}$ This effect can be attributed to the thermalization of the ablated particles arriving at the substrate surface. As a result, the growth of the Ti rich $\mathrm{SrTiO}_{3}$ changes from a coherent to an island growth mode with rising oxygen pressure. A similar transition from a "pseudo 2D" island growth at low pressures (as observed in Sec. V A) to a Stranski-Krastanov type, full 3D island growth at higher pressures has been reported by Shin et al. ${ }^{26}$

Concerning the clearly observed connection between the defect structure and the local conductivity, we can state that for this specific type of samples the defect-rich island boundaries exhibit a significantly increased conductivity compared to the less conductive island centers. Since the only relevant conduction type at room temperature and low voltages in our material is an electronic conduction, the conductivity can be described as

$$
\sigma=e \mu_{e} n_{e}
$$

with the electron charge $e$, the conduction electron mobility $\mu_{e}$, and concentration of conduction electrons $n_{e}$. Hole-type contributions are negligible. An increased conductivity at the island boundaries has therefore to be related to either an increase in $n_{e}$ or an increase in $\mu_{e}$. While $\mu_{e}$ is related to the local band structure, in particular the band bending, $n_{e}$ is determined by the local stoichiometry. Since the conductivity is typically extremely sensitive to stoichiometry variations, we assume that the main part of the conductivity variation between island boundary and center can be attributed to a stoichiometry and therefore $n_{e}$ change, while possible variations $\mu_{e}$ play a secondary role.

In a first approximation, (where point defect chemistry considerations have been extrapolated to a local scale) $n_{e}$ is equal to

$$
n_{e}=2\left[\mathrm{~V}_{\mathrm{O}}^{\prime \prime}\right]+\left[\mathrm{Nb}_{\mathrm{Ti}}^{\prime}\right]-2\left[\mathrm{~V}_{\mathrm{Sr}}^{\prime \prime}\right]
$$

where $\mathrm{V}_{\mathrm{O}}^{*}$ are double positively charged oxygen vacancies, $\mathrm{Nb}_{\mathrm{Ti}}$ ionized, and therefore positively charged dopant atoms and $\mathrm{V}_{\mathrm{Sr}}^{\prime \prime}$ double negatively charged $\mathrm{Sr}$ vacancies. An increased $n_{e}$ at the island boundaries can therefore, be caused by an increase in $\mathrm{V}_{\mathrm{O}}^{*}$ or $\mathrm{Nb}_{\mathrm{Ti}}^{\circ}$ or a decrease in $\mathrm{V}_{\mathrm{Sr}}^{\prime \prime}$. Since we cannot detect any variation in the $\mathrm{Nb}$ content across our film and since a decrease in $\mathrm{Sr}$ vacancies in a $\mathrm{SrTiO}_{3}$ area of increased defect density seems unlikely, we suggest that the increased conductivity of the island boundaries might be related to an increased oxygen vacancy content within these defective regions. While De Souza et al. have reported on oxygen-deficient low angle grain boundar ${ }^{21,32} \mathrm{Jia}$ et al. could directly prove the existence of oxygen-deficient dislocations in $\mathrm{SrTiO}_{3}$ single crystals. ${ }^{22,33}$ Concerning the conductivity distribution Szot and Bihlmayer presented DOS calculations showing a significantly improved conductivity in the direction of such a defect. ${ }^{7}$

The observed high conductivity of the island boundaries can therefore possibly explained by a locally increased density of oxygen deficient extended defects.
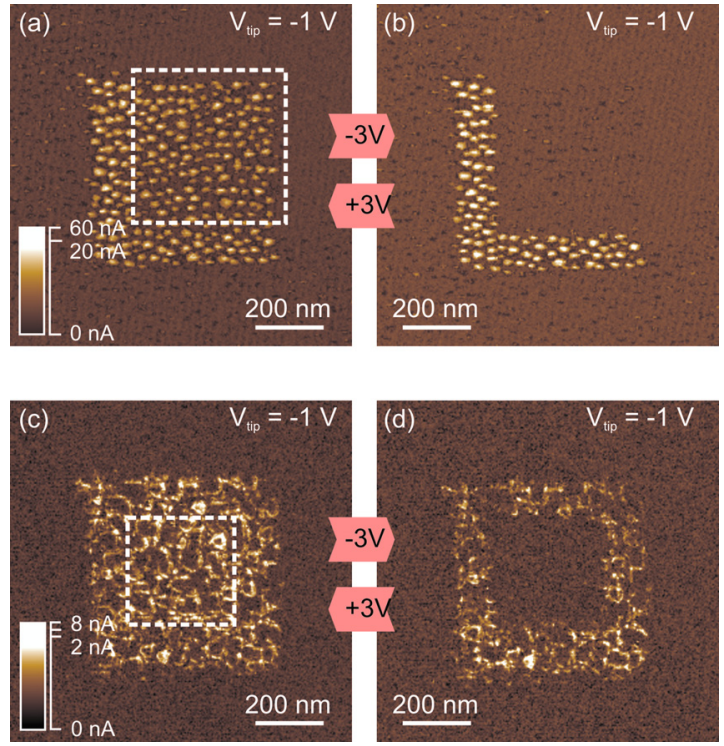

FIG. 7. (Color online) [(a) and (b)] Reversible resistive switching in a 2 at. \% $\mathrm{Nb}$-doped $\mathrm{SrTiO}_{3}$ sample grown in a coherent, 3D way. The conductive AFM current images show an array of conducting units which can be switched between two different resistance states. [(c) and (d)] Reversible resistive switching in a 2 at. \% $\mathrm{Nb}$-doped $\mathrm{SrTiO}_{3}$ sample grown in an island growth mode. The array of conducting rings corresponds to the film's defect structure and can be switched between two different resistance states.

\section{AFM TIP INDUCED SWITCHING}

Conductive AFM is typically used as a passive probing tool to determine the surface structure and local conductivity of a sample as described in the previous sections. In the language of resistive switching this corresponds to a readout measurement that does not change the sample's resistive state. The optimal readout voltage value is reached for a voltage that is sufficient to provide a reasonable current signal and low enough not to change the samples resistivity upon several readout scans. It varies between different sample types and has to be readjusted prior to each measurement.

It is however also possible to use the AFM tip as an active switching tool. ${ }^{7,12}$ If biased with an appropriate switching voltage it can be regarded as a mobile virtual top electrode. In order to ensure good reproducibility special care was taken to avoid topographical and structural changes during switching and to keep the switching voltage as low as possible. Higher voltages result in a drastic alteration of the local surface structure. While Pellegrino et al. ${ }^{34}$ have utilized these tip voltage induced surface changes to realize an AFM controlled surface lithography, they are undesirable in terms of resistive switching because they represent an undefined and irreversible change in the sample. Absolute voltage values of 3-6 V were usually sufficient to change the sample's resistance without modifying its morphology. Similar to the readout voltage, the switching voltages has to be readjusted for each sample as well.

An AFM switching procedure for the 2 at. \% Nb-doped $\mathrm{SrTiO}_{3}$ sample grown in the coherent 3D growth mode, described in Sec. V A, is shown in Figs. 7(a) and 7(b). Since the topography is constant throughout the switching process and identical to the one shown in Fig. 5, only current images 
are shown. Starting with a readout scan at-1 V in part (a), the sample specific conductivity pattern can be seen. In this case it is the previously described short-range-ordered array of well conducting units. A central $500 \times 500 \mathrm{~nm}^{2}$ square of these units appears as a bright and conductive region. (All units outside this square have been previously switched off to allow a better visual contrast.) Following this initial readout scan, the tip was biased with a higher voltage of $-3 \mathrm{~V}$ and scanned across the upper right part of the central square, marked in white (scan not shown here). A second readout scan, that was taken after this switching step and is shown in part (b), reveals, that the switching scan has caused all units within its scanning range to switch off. These units can be switched back into the On state with a switching voltage of $+3 \mathrm{~V}$ and the initial state is reached again. Continuing in this manner of subsequent switching and readout scans a reversible switching between On and Off state can be generated that can be repeated several times. The eventually limiting factor is not the sample but the AFM tip wear off.

On a large scale, the switching effect can be approximated as homogeneous, in the sense that it can be induced in any arbitrarily chosen and shaped area of the sample, without any structural or surface changes. On a local scale, however, it has a substructure that is dictated by the conducting units. The conductivity of these units is changed by roughly three orders of magnitude during switching, varying between a low current level of $0.03 \mathrm{nA}$ in the Off state and a high current level of roughly $10 \mathrm{nA}$ in the On state. The surrounding background region remains poorly conducting throughout the switching procedure and therefore does not contribute to the effect. Moreover, placing the tip permanently above a single unit, local current-voltage scans can be recorded, that reflect the On and Off switching of the respective, individual unit. ${ }^{35}$ According to the discussion in Sec. $\mathrm{V} A$, these regularly arranged resistive switching units might be identified with the center of threading dislocations.

Figures $7(\mathrm{c})$ and $7(\mathrm{~d})$ contains current images of the 2 at. \% Nb-doped $\mathrm{SrTiO}_{3}$ sample grown in an island growth mode, that was presented in Sec. V B. Performing the same switching procedure as with the previous sample, a similar switching behavior can be seen: starting with a well conducting, squared region in part (c), a smaller, central part of that square was scanned with a switching voltage of $-3 \mathrm{~V}$. A subsequent readout scan [part (d)] confirms a successful off switching of that central region. Scanning with $+3 \mathrm{~V}$ revives the conductivity, while scanning with $-3 \mathrm{~V}$ decreases it again. The topography of the sample is again unchanged during the switching process and identical to the one shown in Fig. 6.

While the qualitative switching properties, such as the overall procedure, the polarity and the possibility to switch any designated region are identical to the previously presented sample, the site of resistive switching has been adapted to the second sample's changed defect structure: the defect-rich growth island boundaries contribute to the switching, while the less defective interior remains poorly conducting throughout the process.

The observed nanoscale switching behavior is consistent with a voltage driven oxygen vacancy movement reported in the literature ${ }^{8,7,36-38}$ that induces a local redox-based metal to insulator transition in transition metal oxides. While homogeneous on a larger scale, on a local scale, the oxygen diffusion is preferentially occurring in defect-rich regions of the investigated samples. This can be either explained by the strongly increased density of oxygen vacancies in the defective regions or by their increased mobility within the extended defects, that can possibly act as fast diffusion channels for oxygen vacancies. ${ }^{39}$

\section{CONCLUSION}

In conclusion, we succeeded to grow $\mathrm{SrTiO}_{3}$ films with widely different defect structures using PLD. $\mathrm{SrTiO}_{3}$ thin films, grown in a layer-by-layer grow mode with a Ti/Sr ratio close to 1 exhibit a defect structure and conductivity pattern close to single crystals. Irregularly distributed resistive switching, conducting spots were observed in an insulating matrix and might be identified with exits of dislocations, that extend across the sample and intersect with the surface. For Ti-rich films grown at low pressure, a coherent 3D growth mode was obtained. The significant surface feature of these films is a short-range-order of well-conducting surface hills. These regular arrays of conducting hills can be regarded as single resistive switching units. Both, the size and the distribution of these conducting hills, resemble regular networks of threading dislocations. For Ti-rich films grown at higher pressure in a kinetically more restricted island growth mode, we were able to identify defect-rich island boundaries with conducting ring structures on the surface: each island boundary can be seen as a well conducting region, while the less defective island centers are nonconducting and not involved in the switching process. The observed nanoscale switching behavior is consistent with a voltage driven oxygen vacancy movement that induces a local metal to insulator transition. The transition occurs preferentially in defect-rich regions of the investigated samples containing an increased concentration of oxygen vacancies and acting as easy-diffusionchannels. Our results demonstrate the possibility to modify the local distribution of resistive switching channels by choosing appropriate deposition routes and are thereby a first step toward the realization of defect engineered $\mathrm{SrTiO}_{3}$ thin films.

\section{ACKNOWLEDGMENTS}

We acknowledge K. Szot for his advice during the conductive AFM measurements and XPS measurements, P. Meuffels and C. Makovicka for the preparation of ceramic targets, and K. Shibuya for helpful discussions.

${ }^{1}$ J. Mannhart and D. G. Schlom, http://dx.doi.org/10.1038/430620a (2004). ${ }^{2}$ J. Son, P. Moetakef, B. Jalan, O. Bierwagen, N. J. Wright, R. EngelHerbert, and S. Stemmer, Nature Mater. 9, 482 (2010).

${ }^{3}$ E. Bousquet, M. Dawber, N. Stucki, C. Lichtensteiger, P. Hermet, S. Gariglio, J. M. Triscone, and P. Ghosez, Nature (London) 452, 732 (2008).

${ }^{4}$ A. Ohtomo and H. Y. Hwang, Nature (London) 427, 423 (2004).

${ }^{5}$ L. W. Chang, M. Alexe, J. F. Scott, and J. M. Gregg, Adv. Mater. 21, 4911 (2009).

${ }^{6}$ S. V. Kalinin, B. J. Rodriguez, A. Y. Borisevich, A. P. Baddorf, N. Balke, H. J. Chang, L. Q. Chen, S. Choudhury, S. Jesse, P. Maksymovych, M. P. Nikiforov, and S. J. Pennycook, Adv. Mater. 22, 314 (2010). 
${ }^{7}$ K. Szot, W. Speier, G. Bihlmayer, and R. Waser, Nature Mater. 5, 312 (2006).

${ }^{8}$ R. Waser, R. Dittmann, G. Staikov, and K. Szot, Adv. Mater. 21, 2632 (2009).

${ }^{9}$ D. B. Strukov, G. S. Snider, D. R. Stewart, and R. S. Williams, Nature (London) 453, 80 (2008).

${ }^{10}$ K. Shibuya, R. Dittmann, S. Mi, and R. Waser, Adv. Mater. 22, 411 (2010).

${ }^{11}$ Crystec, http://www.crystec.de/daten/srtio3.pdf

${ }^{12}$ K. Szot, R. Dittmann, W. Speier, and R. Waser, Phys. Status Solidi (RRL) 1, R86 (2007).

${ }^{13}$ W. Frammelsberger, G. Benstetter, J. Kiely, and R. Stamp, Appl. Surf. Sci. 253, 3615 (2007)

${ }^{14}$ J. Fleig and J. Maier, Solid State Ionics 86-8, 1351 (1996).

${ }^{15}$ T. Ohnishi, M. Lippmaa, T. Yamamoto, S. Meguro, and H. Koinuma, Appl. Phys. Lett. 87, 241919 (2005).

${ }^{16}$ T. Ohnishi, K. Shibuya, T. Yamamoto, and M. Lippmaa, J. Appl. Phys. 103, 103703 (2008).

${ }^{17}$ T. Suzuki, Y. Nishi, and M. Fujimoto, Philos. Mag. A 80, 621 (2000).

${ }^{18}$ C. M. Brooks, L. F. Kourkoutis, T. Heeg, J. Schubert, D. A. Muller, and D. G. Schlom, Appl. Phys. Lett. 94, 162905 (2009).

${ }^{19}$ R. Moos and K. H. Haerdtl, J. Am. Ceram. Soc. 80, 2549 (1997).

${ }^{20}$ T. Menke, P. Meuffels, R. Dittmann, K. Szot, and R. Waser, J. Appl. Phys. 105, 066104 (2009).

${ }^{21}$ J. De Souza, J. Fleig, and J. Maier, J. Appl. Phys. 97, 053502 (2005).

${ }^{22}$ C. L. Jia, A. Thust, and K. Urban, Phys. Rev. Lett. 95, 225506 (2005).

${ }^{23}$ R. Münstermann, Ph.D. thesis, RWTH Aachen University, 2006.

${ }^{24}$ A. Stashans and L. Villamagua, J. Phys. Chem. Solids 70, 417 (2009).
${ }^{25}$ Y. Zhu, Acta Mater. 53, 1277 (2005).

${ }^{26}$ J. Shin, S. V. Kalinin, A. Y. Borisevich, E. W. Plummer, and A. P. Baddorf, Appl. Phys. Lett. 91, 202901 (2007).

${ }^{27}$ Y. L. Qin, C. L. Jia, K. Urban, J. H. Hao, and X. X. Xi, J. Mater. Res. 17, 3117 (2002).

${ }^{28}$ C. J. Lu, L. A. Bendersky, K. Chang, and I. Takcuchi, Symposium on Structure-Property Relationships of Oxide Surfaces and Interfaces II held at the 2002 MRS Fall Meeting (Boston, MA, 2003), pp. 37-42.

${ }^{29} \mathrm{~F}$. Baudenbacher, Oberflächenmorphologie und Wachstumsmodus epitaktischer YBa2Cu3O7-Filme (Shaker Verlag, Aachen, 1997).

${ }^{30}$ J. Q. He, E. Vasco, R. Dittmann, and R. H. Wang, Phys. Rev. B 73, 125413 (2006)

${ }^{31}$ G. Rijnders, Ph.D. thesis, Universiteit Twente, 2001.

${ }^{32}$ J. Maier and R. Merkle, Angew. Chem., Int. Ed. 47, 21 (2008).

${ }^{33}$ C. L. Jia, L. Houben, and K. Urban, Philos. Mag. Lett. 86, 683 (2006).

${ }^{34}$ L. Pellegrino, E. Bellingeri, A. S. Siri, and D. Marre, Appl. Phys. Lett. 87, 064102 (2005)

${ }^{35}$ R. Muenstermann, R. Dittmann, K. Szot, S. Mi, C.-L. Jia, P. Meuffels, and R. Waser, Appl. Phys. Lett. 93, 023110 (2008).

${ }^{36}$ J. J. Yang, M. D. Pickett, X. Li, D. A. A. Ohlberg, D. R. Stewart, and R. S. Williams, Nat. Nanotechnol. 3, 429 (2008).

${ }^{37}$ Y. B. Nian, J. Strozier, N. J. Wu, X. Chen, and A. Ignatiev, Phys. Rev. Lett. 98, 146403 (2007).

${ }^{38}$ M. J. Rozenberg, M. J. Sanchez, R. Weht, C. Acha, F. Gomez-Marlasca, and P. Levy, Phys. Rev. B 81, 115101 (2010).

${ }^{39}$ K. Szot, W. Speier, R. Carius, U. Zastrow, and W. Beyer, Phys. Rev. Lett. 88, 075508 (2002). 